\title{
The Osseous Dissipation- Otosclerosis
}

\author{
Anubha Bajaj* \\ Consultant Histopathologist, A.B. Diagnostics, India
}

*Corresponding author: Anubha Bajaj, Consultant Histopathologist, A.B. Diagnostics, A-1, Ring Road, Rajouri Garden, New Delhi 110027, India

\begin{tabular}{|c|c|}
\hline ARTICLE INFO & ABSTRACT \\
\hline Received: 蔧 December 14, 2021 & $\begin{array}{l}\text { Citation: Anubha Bajaj. The Osseous Dissipation- Otosclerosis. Biomed J Sci \& Tech Res } \\
\text { 40(4)-2021. BJSTR. MS.ID.006477. }\end{array}$ \\
\hline
\end{tabular}

\section{Preface}

Otosclerosis is a primary osteodystrophy of the otic capsule or a disorder of bone remodelling incriminating the stapes footplate or bony labyrinth of the inner ear. Otosclerosis literally implies "abnormal hardening of body tissue of the ear". The condition is confined to the middle ear and is associated with anomalous remodelling of bone. Normally dense endochondral layer of bony otic capsule situated within the labyrinth is substituted by irregular, spongy bone. Consequently, fixation of the stapes ensues. Otosclerosis was initially scripted by Antonio Maria Valsalva in 1735 and is additionally designated as otospongiosis. Otosclerosis alters functioning of the middle ear and inner ear and is a predominant contributor to deafness in adults. Otosclerosis may engender significant disability and morbidity due to hearing loss. Antecedent disease detection with cogent therapy is associated with superior outcomes.

\section{Disease Characteristics}

Typically, otosclerosis induces lucent alterations instead of sclerotic bony modifications. Temporal bones are frequently incriminated [1,2]. Otosclerosis appears as an antecedent, adult onset disease. Generally, otosclerosis commences within second decade or third decade although hearing loss commences following the fourth decade. Disease occurrence within children is exceptional. However, the progressive condition demonstrates gradually worsening clinical symptoms and it may be challenging to ascertain precise disease onset $[1,2]$. A female predominance is observed with a female to male proportion of $\sim 2: 1$. Otosclerosis worsens in pregnancy [1,2]. Ethnic predilection demonstrates frequent disease emergence within the Caucasian population. Otosclerosis is exceptionally discerned in Asians or Black population. Familial disease incidence appears in an estimated 50\% instances. Around $85 \%$ instances are bilateral $[1,2]$. Otosclerosis is associated with distinctive phases such as

- Early or active otospongiosis and

- Late or inactive otosclerosis.

Of obscure and multifactorial pathogenesis, otosclerosis may arise due to genetic, viral, inflammatory or autoimmune components $[1,2]$. Preliminary lesions are predominantly comprised of an admixture of histiocytes, osteoblasts and osteocytes wherein osteocytes are an active cellular component. Bony circumscription of pre-existing vascular articulations is resorbed with meliorated microcirculation. Eventually, osteoblasts configure irregular foci of nascent, spongy bone, designated as "blue mantles of Manasse" $[1,2]$.

\section{Disease Pathogenesis}

Of obscure aetiology, otosclerosis is posited to denominate a multifactorial emergence such as

- Anatomical factors wherein fissula ante fenestram is incriminated along with persisting remnants of embryonic cartilage $[3,4]$. 
- Genetic factors of otosclerosis are associated with several loci situated upon chromosomes 6p, 9p, 1q, 3q, 6q, 7q, 15q, $16 \mathrm{q}$ and a contemporary locus upon chromosome $7 q 22.1$. Additionally, diverse genes as type I collagen (COL1A1 gene), transforming growth factor- $\beta 1$ (TGF- $\beta 1$ ) with BMP 2 and BMP4 genes, angiotensin II with AGT M235T and ACE I/D genes may induce otosclerosis [3,4]. Sex hormones, autoimmune reaction, human leucocyte antigen (HLA), inflammatory or regulatory cytokines, parathyroid hormone, parathyroid hormonerelated peptide receptors and oxidative stress may initiate otosclerosis $[3,4]$.

- Hereditary factors wherein an estimated 50\% subjects with otosclerosis demonstrate a family history. Individuals with hereditary otosclerosis are associated with an antecedent disease onset. Majority of instances depict an autosomal dominant mode of inheritance accompanied by reduced penetrance in nearly $\sim 40 \%$ subjects and variable expression of disease $[3,4]$.

- Viral infection is implicated in the emergence of otosclerosis. Ribonucleic acid (RNA) of measles virus may be discerned within footplate of stapes upon ultrastructural examination and immunohistochemistry. Vaccination for measles virus may appear as a safeguard for occurrence of otosclerosis $[3,4]$. Additionally, factors such as menopause, trauma, bone dyscrasia or major surgery may initiate or aggravate otosclerosis [3,4].

\section{Clinical Elucidation}

Otosclerosis is commonly confined to the temporal bone and engenders fixation of footplate of stapes within the oval window. Therefore, inability to transmit sound waves manifests as conductive hearing loss [5,6]. Generally, otosclerosis engenders conductive deafness and appears in association with a normal tympanic membrane. Contingent to focal incrimination of the bony labyrinth, otosclerosis can be asymptomatic or represent with neurosensory decimation [5,6]. Frequent clinical representation of otosclerosis is hearing loss accompanied by tinnitus and vertigo. Gradually progressive bilateral hearing loss is a common symptom which typically commences in one ear and progresses to contralateral ear $[5,6]$. Inability to adequately discern low-frequency sounds as a whisper can emerge as an initial clinical symptom. Hearing may be meliorated in a noisy environment, a feature denominated as "paracusis willisii" which is indicative of conductive deafness. Incriminated subjects adopt a low-volume, monotonous tone of voice.

Extensive otosclerosis is associated with worsening tinnitus. Mild dizziness appears and deteriorates with disease progression, simulating Meniere's disease [5,6]. Otoscopy may be normal or depict minimal modifications. However, around $10 \%$ instances of active otosclerosis or cochlear otosclerosis are accompanied by enhanced vascularity of the promontory which can be discerned through the tympanic membrane and is designated as "Schwartze sign" $[5,6]$. Of indolent clinical course, otosclerosis manifests with frequently bilateral, conductive hearing loss although sensorineural or mixed deafness may ensue. Hearing loss can be exacerbated in pregnant subjects $[5,6]$. Otosclerosis is comprised of distinctive subtypes denominated as

- Fenestral or stapedial variant which constitutes of a majority $(\sim 80 \%)$ of instances and incriminates the oval window along with stapes footplate. Frequently, conductive hearing loss is associated with thickening and fixation of stapes [5,6].

- Retrofenestral or cochlear subtype which is composed of nearly $20 \%$ instances. Incriminated cochlea is associated with demineralization of cochlear capsule. Sensorineural hearing loss emerges, possibly due to uncertain mechanisms $[5,6]$. Retrofenestral otosclerosis is usually accompanied by fenestral variant and the conditions are contemplated to represent a disease continuum $[5,6]$.

\section{Histological Elucidation}

Upon gross examination, foci of otosclerosis appear as chalky white, greyish or yellowish. Active centric zone and rapid disease progression is associated with reddish lesions due to enhanced vascularity. Upon macroscopic examination of dissected tissue, specimens of un-altered head and crura of stapes are obtained [5,6]. Microscopically, foci of initial bone resorption and circumscription of vascular articulations by cellular, fibrovascular tissue are observed. Subsequently, deposition of immature bone, perpetual bone resorption and remodelling is exemplified. Gradually, deposition of bone, enhanced collagen and decimated ground substance ensue with consequent emergence of densely sclerotic bone demonstrating prominent cement lines [5,6]. Dense, endochondral layer of otic capsule appears to be constituted of spongy bone. Immature, active lesions of otosclerosis are imbued with abundant bone marrow, vascular spaces and numerous osteoblasts admixed with osteoclasts. Significant quantities of cement substance are deposited within the lesion [5,6]. Mature foci of otosclerosis are mildly vascular and composed of abundant bone and fibrillar substance with minimal cementum. Localized foci of bone remodelling and bone resorption of the otic capsule are followed by subsequent bone deposition.

Otoclerosis predominantly arises due to replacement of normal bone with sclerotic or spongiotic bone [5,6]. Osteolytic osteocytes are configured upon growing perimeter of the lesion. Sheets of connective tissue displace the bone. Delayed stage of otosclerosis depicts dense, sclerotic bone associated with zones of previous 
bone resorption. Consequently, disorganized bone is articulated, osteocytes are quantifiably enhanced and expansive bone marrow spaces are incorporated with vascular articulations and connective tissue [5,6]. Bone marrow spaces are eventually substituted by dense, sclerotic bone imbued with narrow vascular articulations and minimal Haversian canal system [5,6]. Preliminary and delayed stage of otosclerosis occurring within a singular temporal bone engenders pleomorphism. Antecedent lesions may adjoin fissula ante fenestram and expand through abutting vascular channels $[5,6]$. Majority of lesions are confined to anterior oval window and appear associated with calcification of annular ligament or incrimination of stapes, thereby engendering conductive hearing loss [5,6] (Figures 1-8).

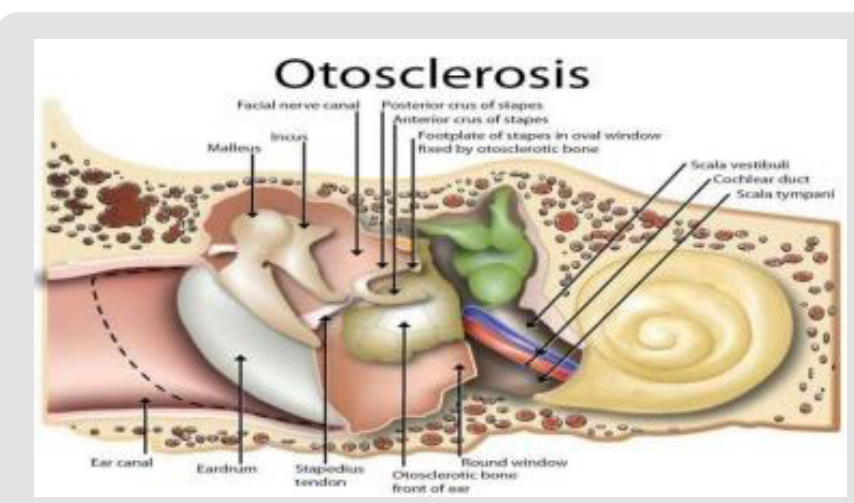

Figure 1: Otosclerosis exhibiting sclerotic bone of footplate of stapes and bony labyrinth of inner ear [9].

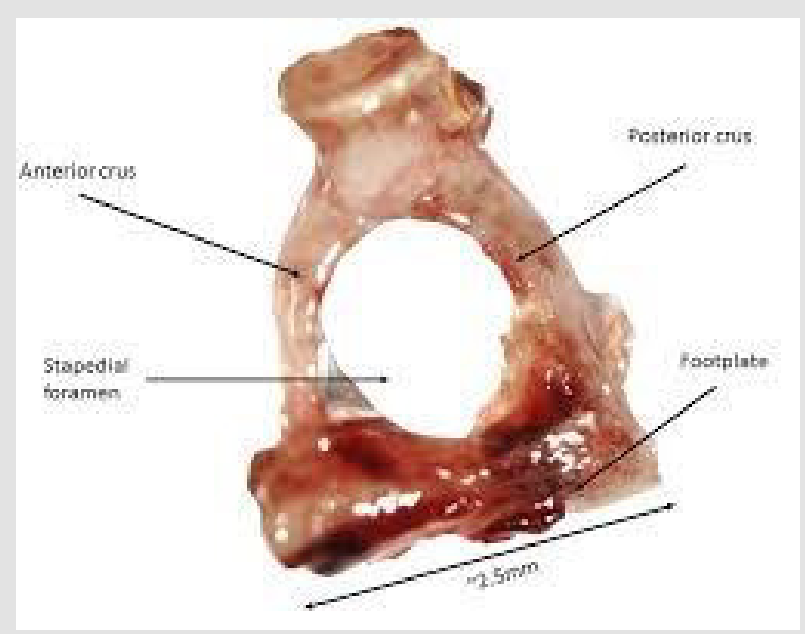

Figure 2: Otosclerosis enunciating spongiotic and sclerotic bones replacing foot plate of stapes [10].

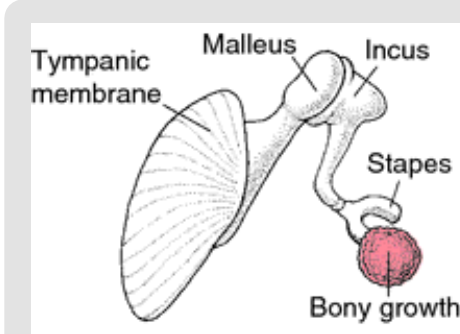

Otosclerosis at the anterior footplate

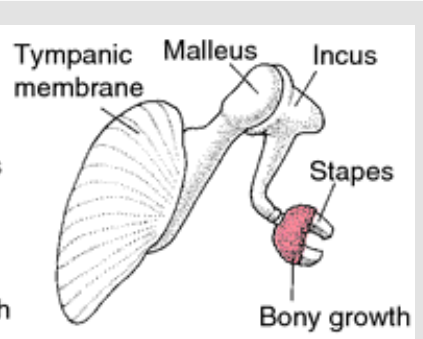

Otosclerosis at the junction of the incus and stapes
Figure 3: Otosclerosis exemplifying bony growth within footplate of stapes and spongiotic bone at the junction of incus and stapes [11].

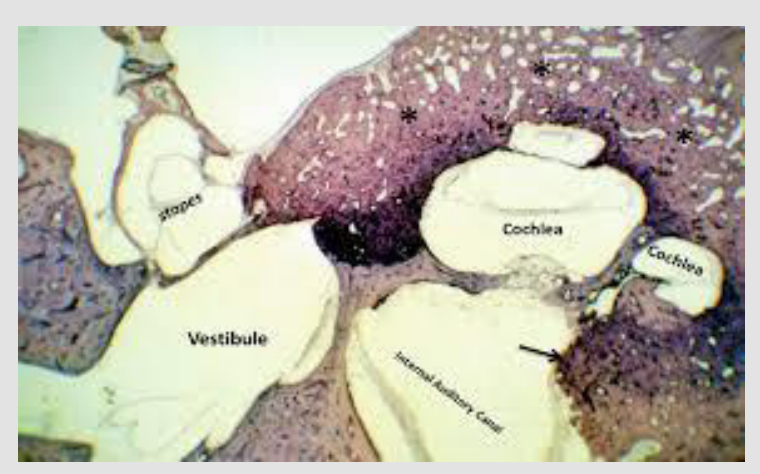

Figure 4: Otosclerosis exemplifying bony sclerosis at footplate of stapes with an admixture of spongiotic bone, osteocytes and minimal Haversian system [12].

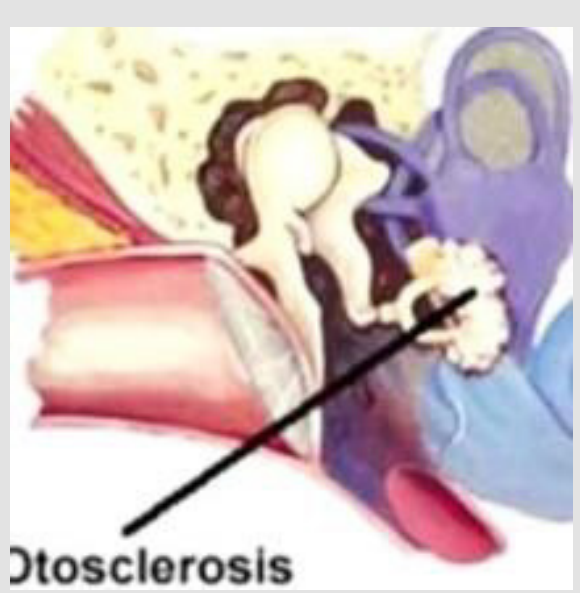

Figure 5: Otosclerosis delineating bony sclerosis of footplate of stapes with configured spongiotic bone and associated conductive hearing loss [13]. 


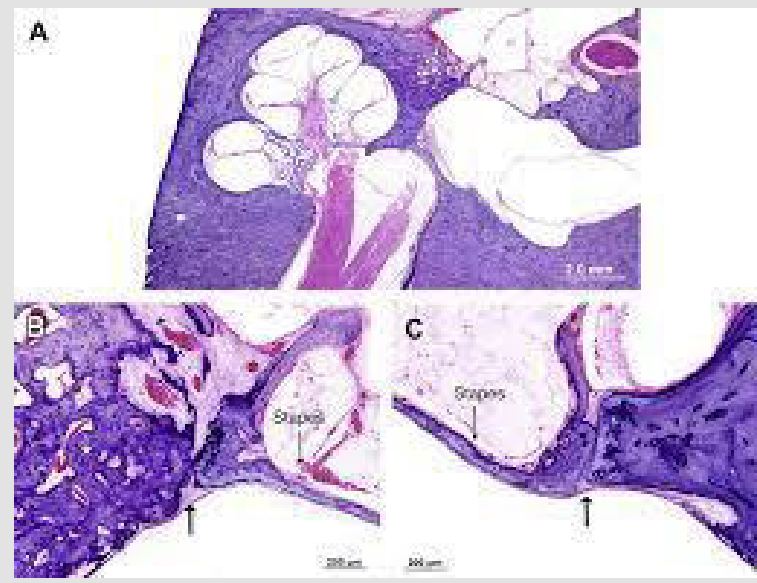

Figure 6: Otosclerosis demonstrating bony sclerosis and spongiotic bone replacing footplate of stapes with intermingled osteocytes and fibro-connective tissue [14].

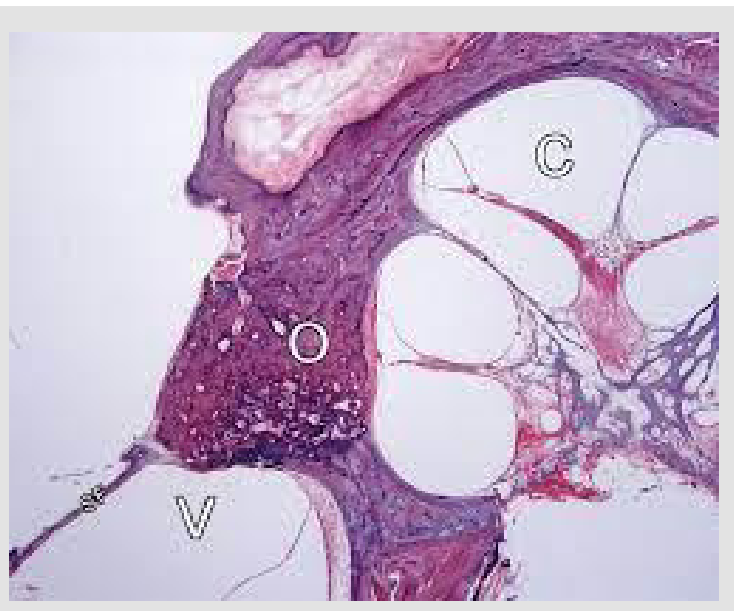

Figure 7: Otosclerosis displaying spongy and sclerotic bone replacing footplate of stapes intermingled with abundant osteocytes, osteoblasts and fibro-connective tissue [15].

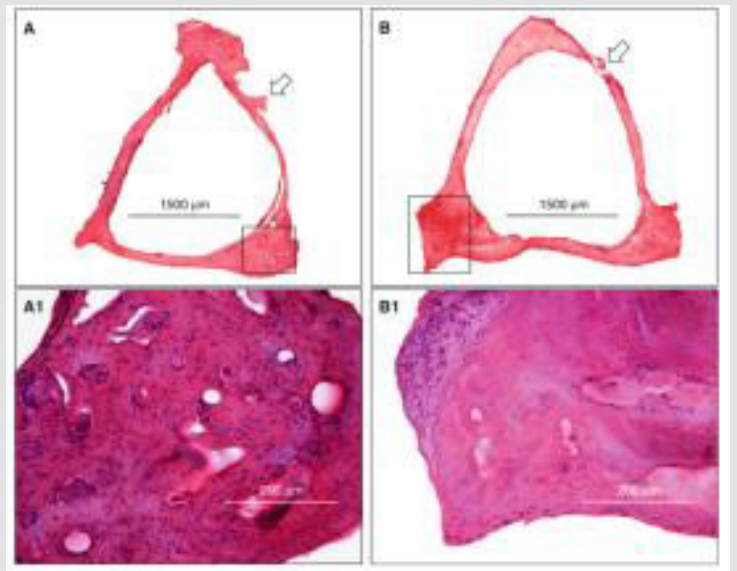

Figure 8: Otosclerosis exhibiting bony sclerosis of the inner ear labyrinth with several osteocytes, decimated Haversian canal and fibroconnective tissue [16].

\section{Differential Diagnosis}

Otosclerosis requires a segregation from conditions such as

- Osteogenesis imperfecta where otic capsule demonstrates lucencies simulating otosclerosis. Osteogenesis imperfecta is associated with non deformed, fragile bones and blue sclera. Severe variants of osteogenesis imperfecta are devoid of an organized bone trabecular pattern. Crowding of osteocytes within bone occurs due to decimated collagen synthesis. Enlarged foci of woven bone are delineated. Minimally severe instances depict crowding of osteocytes associated with attenuated lamellar bone [2,4].

- Paget's disease of bone predominantly occurs in elderly individuals and demonstrates bony expansion. Acute stage is primarily composed of woven bone admixed with focal, mosaic configuration of lamellar bone associated with irregular cement lines. Osteoclasts are preponderant upon the bone surface. Osteoclasts appearing within osteolytic phase may depict around $\sim 100$ nuclei $[2,4]$. Chronic stage is comprised of thickened bony trabeculae and thick bones which are imbued with finely fibrotic bone marrow $[2,4]$.

- Osteoradionecrosis arises due to vascular injury with consequent emergence of bony ischemia. Osteoradionecrosis is associated with distinctive phases denominated as

- Pre-fibrotic phase demonstrating foci of chronic inflammatory cell exudate

- $\quad$ Phase of organized fibrosis predominantly displaying aberrant fibroblastic activity along with an inadequately organized bone matrix. Aforesaid regions appear adjacent to foci of aging fibroblasts disseminated within a minimally cellular, fibrotic, densely sclerotic matrix $[2,4]$.

- Fibro-atrophic phase is composed of dense hyalinization and fibrosis along with absence of bone marrow cells. Focal, reactive stratified squamous epithelium may layer a fistulous tract. Patchy, secondary infiltration of chronic inflammatory cells may occur. Lesions may display necrotic or sclerotic bone along with empty osteocyte lacunae. Fibrosis of bone marrow is admixed with irregular bony trabeculae devoid of osteocytes within the lacuna. Foci of bone marrow necrosis may arise due to emergence of a preliminary stage of oedematous, fibromyxoid disease [2,4]. Additionally, otosclerosis requires a segregation from conditions which are associated with conductive deafness such as serous otitis media, adhesive otitis media, congenital fixation of the stapes, Meniere disease, tympanosclerosis, attic fixation of head of the malleus or ossicular discontinuity [2,4]. 


\section{Investigative Assay}

Upon otoscopy, features indicative of otosclerosis are minimal to absent. However, severe instances with cochlear incrimination can engender hyperaemia of the cochlear promontory, a feature designated as "Schwartze sign" [7,8]. Otosclerosis can be appropriately investigated with the tuning fork and demonstrates a negative Rinne's test. Weber's test appears lateralized to the ear and is indicative of a severe conductive hearing loss $[7,8]$. Pure tone audiometry demonstrates low frequency loss of air conduction with normal bone conduction. Pure tone audiometry exhibits a characteristic decimation of bone conduction, especially at higher frequencies $[7,8]$. Mixed hearing loss can be observed wherein preliminary otosclerosis is associated with normal tympanometry $[7,8]$. High resolution computerized tomography (CT) of temporal bone is optimal in discerning otosclerosis besides recognizing and segregating associated causes of deafness. An estimated $80 \%$ instances depict fenestral foci which are situated anterior to the oval window. Also, thickened stapedial footplate and incriminated round window can be discerned, features which regulate appropriate therapy $[7,8]$. Retrosternal focus emerges as a "double halo" sign, a feature encountered with cochlear otosclerosis. Otosclerosis can be appropriately graded with CT $[7,8]$.

Pertinent imaging depicts representations such as

- Fenestral otosclerosis which is the commonest variant and demonstrates site of incrimination just anterior to oval window while configuring a miniature cleft denominated as fissula ante fenestram. Besides, bony overgrowth may engender fixation of the stapes $[7,8]$.

- Retrofenestral otosclerosis incriminates the niche of round window and usually accompanies fenestral disease. Nevertheless, isolated round window otosclerosis may be occasionally discerned. Predominant site of disease emergence appears as focal or circumferential bone circumscribing the cochlea. Circumferential bony implication engenders a "fourth turn" or "double ring" sign [7,8]. Imaging features are contingent to phase of the disease and appear as

- Otospongiotic phase composed of demineralization and configuration of spongy bone. Pertinent phase manifests as decimated attenuation or lucent zone within homogeneously dense perimeter of otic capsule $[7,8]$.

- Otosclerotic phase is associated with enhanced attenuation within disease specific region. As it may be challenging to discern otosclerotic bone from encompassing normal bone, features such as thickness of otic capsule or aberrant convexity of contour of otic capsule cortex anterolateral to anterior margin of the oval window may aid the distinction $[7,8]$. Severe instances are associated with comprehensive impaction of oval window or round window with a dense, bony plate associated with complete fixation of stapes $[7,8]$. Thin-slice computerized tomography (CT) through the temporal bone is a preferred imaging modality for adequately exemplifying anatomy of inner ear and emergent, subtle, preliminary modifications of otosclerosis. Upon computerized tomography, otosclerosis is categorized as

- Grade I which is singularly comprised of fenestral, spongiotic or sclerotic lesions which appear as thickened footplate of stapes along with decalcified, narrowed or enlarged round window or oval window $[7,8]$.

- Grade 2 is constituted of patchy, localized cochlear disease along with the presence or absence of fenestral otosclerosis and is subdivided into grade $2 \mathrm{~A}$ which implicates basal cochlear turn grade $2 \mathrm{~B}$ which implicates middle or apical cochlear turn grade $2 \mathrm{C}$ which implicates basal turn and middle or apical cochlear turn $[7,8]$.

- $\quad$ Grade 3 is comprised of diffuse, confluent involvement of cochlea and otic capsule along with the presence or absence of fenestral involvement $[7,8]$. Magnetic resonance imaging (MRI) of retrofenestral otosclerosis exhibits a distinctive pericochlear and perilabyrinthine soft tissue intensity upon T1 weighted imaging with contrast enhancement. Besides, enhanced signal intensity upon T2 weighted imaging may be observed $[7,8]$.

\section{Therapeutic Options}

Medical management of otosclerosis is required to circumvent or arrest disease progression. However, optimal, efficacious medical therapy which alleviates otosclerosis remains lacking. Administration of sodium fluoride in order to decimate disease progression is debatable [7,8]. Bisphosphonates are antiresorptive and induce osteoclastic apoptosis. Contemporary bisphosphonates, commonly employed to treat otosclerosis, appear promising $[7,8]$. Bilateral hearing aids can be utilized singularly or in combination with diverse therapies $[7,8]$. Fenestral otosclerosis can be appropriately treated with stapedectomy along with the employment of stapes prosthesis. Optimal and recommended surgical treatment of otosclerosis is stapedotomy or stapedectomy or correction of fixation of foot plate of stapes. Surgical intervention is commonly followed by implantation of prosthesis $[7,8]$. Therapeutic outcomes of surgical intervention in otosclerosis appear to be superior, regardless of surgical manoeuver adopted $[7,8]$. Though surgical intervention is beneficial, hearing aids may be required in certain subjects following surgery $[7,8]$. Untreated otosclerosis may engender significant hearing loss although complete deafness is uncommon $[7,8]$. 
Although infrequent, surgical intervention for treating otosclerosis can induce comprehensive sensorineural deafness within the operated ear. Surgical manoeuvers may induce facial nerve injury or tinnitus. An unpleasant taste may arise for a brief duration $[7,8]$. Prosthetic implantation is associated with granulomatous inflammation and erosion or necrosis of the incus $[7,8]$. Following surgical intervention, an estimated $90 \%$ subjects demonstrate significant amelioration of hearing ability. Hearing remains unaltered or declines in occasional instances $[7,8]$. Laser techniques and vein grafting is associated with superior outcomes. Additionally, reoccurrence of conductive hearing loss may ensue due to displacement of prosthetic implant. Original position of prosthesis is altered on account of collagen contraction within the neo-membrane configured between prosthesis and bony labyrinth $[7,8]$. Revision surgery may exhibit ambiguous outcomes and is usually adopted for treating associated clinical symptoms as facial nerve palsy, persistent vertigo or failure of meliorated hearing $[7,8]$. Nevertheless, revision surgery may be accompanied by inferior outcomes $[7,8]$. Strict monitoring is required in subjects with a family history of otosclerosis. Hearing loss in pregnant subjects may appear due to otosclerosis [7-16].

\section{References}

1. Zafar N, Jamal Z, Moien AB Khan. "Otosclerosis" Stat Pearls International, Treasure Island, Florida.

2. Rudic M, Keogh I, R Wagner, E Wilkinson, N Kiros, et al. (2015) The pathophysiology of otosclerosis: Review of current research. Hear Res 330(Pt A): 51-6.

ISSN: 2574-1241

DOI: $10.26717 /$ BJSTR.2021.40.006477

Anubha Bajaj. Biomed J Sci \& Tech Res

(c) $(9)$ This work is licensed under Creative Commons Attribution 4.0 License

Submission Link: https://biomedres.us/submit-manuscript.php
3. Viza Puiggrós I, Granell Moreno E, Carlos Calvo Navarro, Mercè Bohé Rovira, César Orús Dotu, et al. (2020) Diagnostic utility of labyrinth capsule bone density in the diagnosis of otosclerosis with high resolution tomography. Acta Otorrinolaringol Esp 71(4): 242-248.

4. Bittermann AJ, Wegner I, Bo Jan Noordman, Robert Vincent, Geert J M G van der Heijden, et al. (2014) An introduction of genetics in otosclerosis: a systematic review. Otolaryngol Head Neck Surg 150(1): 34-39.

5. Schrauwen I, Weegerink NJ, E Fransen, C Claes, R J E Pennings, et al. (2019) A new locus for otosclerosis, OTSC10, maps to chromosome 1q41-44. Clin Genet 79(5): 495-497.

6. Crompton M, Cadge BA, Joanna L Ziff, Andrew J Mowat, Robert Nash, et al. (2019) The Epidemiology of Otosclerosis in a British Cohort. Otol Neurotol 40(1): 22-30.

7. Arli C, Gulmez I, Elif Tuğba Saraç, Şemsettin Okuyucu (2020) Assessment of inflammatory markers in otosclerosis patients. Braz J Otorhinolaryngol 86(4): 456-460.

8. Morrison AW (1967) Genetic factors in otosclerosis. Ann R Coll Surg Engl 41(2): 202-237.

9. Image 1 Courtesy: Medic for you.

10. Image 2 Courtesy: UCL discovery.

11. Image 3 Courtesy: Medical dictionary.

12. Image 4 Courtesy: Springer link.

13. Image 5 Courtesy: Health Jade.

14. Image 6 Courtesy: Otolaryngology clinics of North America.

15. Image 7 Courtesy: Plural publishing.

16. Image 8 Courtesy: Research gate.

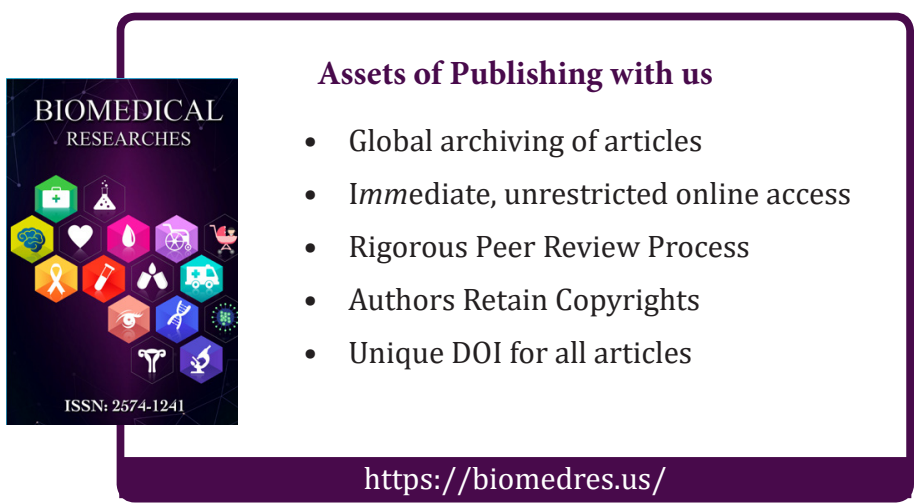

